\title{
STRATEGIES OF GUIDELINES IMPLEMENTATION FOR THE BREAST CANCER EARLY DETECTION IN BRAZIL
}

Sandra Gioia'1,2,3, Lindsay Krush²,4, Paul Goss²,4, Silvio Fernandes³

${ }^{1}$ State Secretariat of Health - Rio de Janeiro (RJ), Brazil.

${ }^{2}$ Global Cancer Institute - Boston (MA), USA.

${ }^{3}$ Sirio Libanes Hospital - São Paulo (SP), Brazil.

${ }^{4}$ Massachusetts General Hospital - Boston (MA), USA.

Early detection actions, according to the recommendations of the Ministry of Health (MH), can increase its effectiveness, and measures are needed to increase the adherence of health professionals and the population. This research project has as general objective to identify strategies of guidelines implementation for the breast cancer early detection that can be used in Brazil. In relation to the specific objectives, they propose to: identify, in the specialized literature, studies on the difficulties and the strategies of implementation of the guidelines for the early detection of breast cancer in the health systems of different countries; to evaluate the applicability of the results found in the Brazilian context; recommend priority actions within the strategies for implementing the guidelines to the organizations responsible for these processes in the field of public health. A systematic review was performed using the Supporting Policy Relevant Reviews and Trial (SUPPORT) tools to aid in the structuring of searches and data analysis. The databases used were PubMed/MEDLINE, Cochrane Library, VHL/LILACS and EMBASE from January 1, 2008 to May 1, 2018. Inclusion criteria: year and period of publication; availability of the full systematic review article in English, Portuguese, French and Spanish; and the use of descriptors. The study of nine selected systematic reviews identified successful actions in developed and developing countries. Actions should, if possible, encompass multiple components, including: organizational change of the system: foster leadership committed to the implementation of guidelines, better governance of health services close to the target audience and flexible schedules, mobile mammography, patient navigation program; in professionals' practice: engagement of breast cancer specialists in primary care to optimize the training of health professionals and users; in the use of health services by the users: national mass publicity campaign, involving multiple MH actors, health secretariats, civil and medical organizations. 